\title{
MICROBIAL ACTIVITY AND COMMUNITY DIVERSITY IN TOBACCO RHIZOSPHERIC SOIL AFFECTED BY DIFFERENT PRE-CROPS
}

\author{
LI, X. ${ }^{1,2}-$ ZHANG, X. ${ }^{1}-\mathrm{HE}, \mathrm{G} .{ }^{3}-\mathrm{XU}, \mathrm{N}^{4}{ }^{4}-\mathrm{SUN}, \mathrm{M}^{5}{ }^{5}$ ZHANG, $\mathrm{H}^{2}{ }^{2}-\mathrm{ZHAO}, \mathrm{Y}^{6^{*}}-\mathrm{YUE}, \mathrm{B} .{ }^{1}-$ \\ SUN, G. ${ }^{1 *}$ \\ ${ }^{1}$ College of Life Science, Northeast Forestry University \\ No.26 Hexing Road, Harbin, Heilongjiang 150040, China \\ ${ }^{2}$ College of Resources and Environment, Northeast Agricultural University \\ Harbin 150030, China \\ ${ }^{3}$ Tobacco Research Institute of Mudanjiang, Mudanjiang 157011, China \\ ${ }^{4}$ Natural Resources and Ecology Institute, Heilongjiang Academy of Sciences \\ Harbin 150040, China \\ ${ }^{5}$ Institute of Crop breeding, Heilongjiang Academy of Agriculture Sciences \\ Harbin 150040, China \\ ${ }^{6}$ School of Forestry, Northeast Forest University, Harbin 150040, China \\ *Corresponding authors \\ e-mail: zhaoys1957@163.com (Zhao,Y.); sungy@vip.sina.com (Sun, G.)
}

(Received $7^{\text {th }}$ Nov 2016; accepted $6^{\text {th }}$ Apr 2017)

\begin{abstract}
In this study, we used both culture-dependent physiological profiling and culture-independent DNA-based approaches to characterize the bacterial communities of tobacco rhizospheric soils affected by different pre-crops (soybean, maize and tobacco). Using Biology-Eco plates, we found that the bacterial metabolic activity in soybean-tobacco and maize-tobacco rotation soils were higher than in tobacco monocropping soil. Across all soil samples, bacterial communities were dominated by Proteobacteria, Acidobacteria and Actinobacteria at the phylum level. However, the diversity and composition of the bacterial communities varied significantly between tobacco rotations and monocropping soil. The estimated bacterial diversity (Shannon diversity index) was higher in the maize-tobacco and soybean-tobacco soils than in tobacco monocropping soil. The populations of Proteobacteria, Acidobacteria, Actinobacteria and Bacteroidetes were found in variable proportions, depending on the different pre-crops. The highest percentages of Proteobacteria, Actinobacteria and Bacteroidetes were found in soybean-tobacco soil, whereas Acidobacteria occurred at higher percentages in tobacco monocropping soil. Collectively, crop rotation influenced soil biodiversity by change of composition and abundance of individual species, and soils under cereal-tobacco rations had higher bacterial activity and diversity than soils under tobacco monocropping.
\end{abstract}

Keywords: soybean, maize, monocropping, rotation, bacterial community structure, bacterial community function

\section{Introduction}

Microorganisms are ubiquitous in the environment and play an essential role in the biogeochemical cycles that sustain all life on Earth (Zarraonaindia et al., 2013; Su et al., 2012). Soil microbial communities are responsible for soil structure maintenance, 
organic matter decomposition, nitrogen fixation, the breakdown of toxic compounds, and inorganic compound transformation (Dick, 1992; Nannipieri et al., 2003). Previous studies have shown that the community structure, abundance and activity of soil microorganisms can be affected by various physical disturbances in the soil (Dick, 1992; Lienhard et al., 2013). Thus, land-use management practices, including crop rotation and tillage (Quadros et al., 2012), fertilizer regime (Qiu et al., 2012), irrigation (Qiu et al., 2012), and continuous cropping (Li et al., 2010) are logical case studies for soil microbial diversity studies.

Tobacco (Nicotiana tabacum L.) is among the most important economic crops in the world, and one million acres of tobacco are planted each year in China (Hecht et al., 1984). Tobacco quality is determined by genetic factors, environmental factors and cultivation measures. Cereal-tobacco rotation is the dominant practice in the northeast of China. Soybean (Glycine max L.) and maize (Zea mays L.) are the major cereal crops grown most often in rotation with tobacco. However, research on the relative effects of the preceding crops on the soil quality of subsequent tobacco in cereal-tobacco rotation systems is rare. It is well known that rotation improves soil quality, including soil biota structure, organic matter content and moisture retention capacity (Ponge et al., 2013). Soils under crop rotation with a high input and diversity of organic materials contain high microbial biomass content and enzyme activity compared with monoculture soils (Trasar-Cepeda et al., 2008). Many studies have found that crop sequence plays a major role in soil C retention (Morari et al., 2006; Varvel, 2008; Wright and Hons, 2005). In particular, the effects of preceding crops on subsequent crops can relate to residual nutrients and water as well as to disease control (Kirkegaard et al., 2008). The decomposition rate of plant debris was found to be mainly governed by the $\mathrm{C} / \mathrm{N}$ ratio. There is growing evidence that the variety of pre-crops can become factors in the microbial population and enzymes in the soils for subsequent crops.

In this study, two different techniques (Biolog-Eco and 454 pyrosequencing) for estimating the soil bacterial community were adopted. To build a sustainable crop-tobacco rotation sequence, the objective was to investigate the bacterial community structure and function of subsequent tobacco in soils in response to three preceding crops (soybean, maize and tobacco) and to observe differences among bacterial communities.

\section{Materials and Methods}

\section{Field plots and sample collection}

Soil sampling was performed in September 2011, at Tobacco Research Institute of Mudanjiang, Ningan city of Heilongjiang province (latitude, $44^{\circ} 85^{\prime} \mathrm{N}$; longitude, $129^{\circ} 60^{\prime} \mathrm{E}$, Northeast China). The average annual temperature in the region is $4^{\circ} \mathrm{C}$. The mean annual precipitation is $427.50 \mathrm{~mm}$, and the evaporation is $1635 \mathrm{~mm}$ per year. The soil is classified as a river silt soil. Selected soil properties were as follows: $\mathrm{pH}$ 6.85; organic matter $27.70 \mathrm{~g} \cdot \mathrm{kg}^{-1}$; total nitrogen $1.90 \mathrm{~g} \cdot \mathrm{kg}^{-1}$; total phosphorus $1.62 \mathrm{~g} \cdot \mathrm{kg}^{-1}$; total potassium $1.61 \mathrm{~g} \cdot \mathrm{kg}^{-1}$; available nitrogen $86.50 \mathrm{mg} \cdot \mathrm{kg}^{-1}$; available phosphorus $36.40 \mathrm{mg} \cdot \mathrm{kg}^{-1}$; available potassium $300.00 \mathrm{mg} \cdot \mathrm{kg}^{-1}$.

The experimental design consisted of three blocks. Each block was divided into three plots representing the three plantation systems. Each plot comprised 8 rows that were $6.00 \mathrm{~m}$ long and $8.80 \mathrm{~m}$ wide; thus, each was $52.80 \mathrm{~m}^{2}$ in size. Treatments levels included (1) tobacco monocropping, (2) soybean-tobacco rotation and (3) maize-tobacco rotation. The cultivars of tobacco, soybean and maize were Longjiang 911, Heinong 34 and Kenfeng 1, respectively. Soil samples were collected from all 
plots, three individual rhizosphere soils randomly collected for each treatment in a plot. Tobacco rhizosphere soils, which adhered to the roots (Nazih et al., 2001), were collected by shaking the soil off the roots. After removal of the vegetation, roots, and stone $(>2 \mathrm{~mm})$, samples were placed into sterile centrifuge tubes under ice and transported to the laboratory within 24 hours. Finally, samples were stored at $4^{\circ} \mathrm{C}$ prior to microbial functional (Biolog ${ }^{\mathrm{TM}} \mathrm{ECO}$ technic) and structural (454 pyrosequencing) analyses.

\section{Substrate utilization patterns and data analysis}

Substrate utilization patterns were measured using Biolog ${ }^{\mathrm{TM}}$ ECO plates (BIOLOG, Inc.). A $10^{-1}$ microbial suspension was prepared by suspending $10 \mathrm{~g}$ of fresh soil in 100 $\mathrm{mL} 0.85 \% \mathrm{NaCl}$ solution. The slurry was processed with a Vortex mixer for $1 \mathrm{~min}$ at maximum speed and centrifuged for $10 \mathrm{~min}$ at $500 \times \mathrm{g}$ (Rutgers et al. 2006). Tenfold serial dilutions were performed, and $150 \mu \mathrm{L}$ of the $10^{-3}$ dilutions were pipetted into microplates using an 8-channel micropipette. Microplates were incubated at $28^{\circ} \mathrm{C}$ for $216 \mathrm{~h}$. The color development at $\mathrm{OD}_{578} \mathrm{~nm}$ was read for each well at $24-\mathrm{h}$ intervals. Negative values were set to zero. The average well color development (AWCD) value of the Biolog data was calculated for each sample at each time point by dividing the sum of the optical density data by 31 (number of substrates), as described by Garland (1996).

The 96-h data were used to measure the functional and species diversity of the soil microbial community, and the following parameters were calculated using the equations below:

Shannon-Wiener index

Simpson index

McIntosh index

$$
H=-\sum P i \times \ln (P i)
$$

where $N i$ is the relative OD in each carbon source well, and $P i$ is calculated by subtracting the control from the absorbance of each substrate and then by dividing this value by the total color change recorded for all 31 substrates.

\section{DNA extraction, amplification of $16 S$ rRNA genes, and pyrosequencing}

Total microbial community DNA was isolated from $0.25 \mathrm{~g}$ of soil per sample using soil DNA extraction kit (Omega Bio-Tek, Atlanta, USA). The extracted DNA was examined following electrophoresis in 1\% agarose gel, and the DNA was normalized to the same concentration prior to amplification. A $\sim 455 \mathrm{bp}$ region of the $16 \mathrm{~S}$ rRNA gene covering the V1-V3 region was selected to construct the community library through tag pyrosequencing. The V1-V3 region was amplified using the universal primers $8 \mathrm{~F}$ (5'-AGAGTTTGATCCTGGCTCAG-3') and 533R (5'-TTACCGCGGCTGCTGGCAC-3') containing the A and B sequencing adaptors (454 Life Sciences). The PCR mixture (final volume, $50 \mu \mathrm{L}$ ) contained $5 \mu \mathrm{M}$ of each primer, $\sim 5 \mathrm{ng}$ of template DNA, $5 \times$ FastPfu PCR buffer, and $2.5 \mathrm{U}$ of FastPfu DNA Polymerase (MBI, Fermentas, USA). The amplification conditions consisted of an 
initial denaturation at $95^{\circ} \mathrm{C}$ for 2 min and 25 cycles of denaturation at $95^{\circ} \mathrm{C}$ at $30 \mathrm{~s}$, annealing at $55^{\circ} \mathrm{C}$ for $30 \mathrm{~s}$, and extension at $72^{\circ} \mathrm{C}$ for $30 \mathrm{~s}$, followed by a final extension period at $72^{\circ} \mathrm{C}$ for $5 \mathrm{~min}$. During amplification, negative control reactions lacking template DNA were also performed to check for experimental contamination. The amplicons were then purified once by gel electrophoresis/isolation and twice more using the Wizard SV Geland PCR Clean-Up System (Promega, Madison, Wisconsin, USA). Next, 454 pyrosequencing was conducted on a Roche massively parallel 454 GS-FIX sequencer according to standard protocols.

\section{Pyrosequencing and data analysis}

Pyrosequencing flowgrams were converted to sequence reads using the MOTHUR software (http://www.mothur.org) and analyzed. The acquired sequences were filtered by evaluating data quality and removing primers and barcodes. Sequences were filtered by the following methods: (1) selecting sequences that contained the barcode and forward primer and eliminating sequences with even a single base pair; (2) removing sequences shorter than $150 \mathrm{bp}$, with ambiguous base pairs, or with more than two wrong matches in the primer; and (3) eliminating barcodes and forward primers. After filtering, the effective sequences were clustered into operational taxonomic units (OTUs) based on phylum, class, order, family, genus and species levels using MOTHUR.

\section{Data analysis}

To detect OTUs, optimized sequences were reduced to $300 \mathrm{bp}$ in length and compared using silva108. Rarefaction curves based on an identified OTU, the species richness estimator Chao 1, were generated for each sample using MOTHUR. The taxonomic assignment of sequences was performed using the Ribosomal Database Project (RDP) classifier (minimum confidence of $80 \%$ ). After phylogenetic allocation of the sequences to the phylum and genus levels, the relative abundance of a given phylogenetic group was set to the number of sequences per sample. Hierarchical cluster (Heatmap) analyses were generated in MOTHUR using the gplots package of R. We used SPSS for windows (version 19) to test for significance $(P<0.05)$ between treatments of relative abundances, Alpha diversity and richness of bacterial communities were analyzed using Duncan post-hoc test at $95 \%$ confidence level. The Duncan test method was conducted for multiple comparisons to assess the significance level of substrate utilization among treatments.

\section{Results}

\section{Community level physiological profile}

As expected, the average well color development (AWCD) increased with incubation period (Fig. 1). The soybean-tobacco rotation consistently exhibited the highest AWCD at all periods, followed by maize-tobacco rotation. There was no significant difference in soil bacterial functional diversity between the soybean-tobacco and maize-tobacco treatments. The tobacco monocropping treatment constantly had the lowest AWCD throughout all period. The soil bacterial functional diversity index $(H, D$ and $S)$ was significantly affected by the pre-crop treatment (Table 1). Among rotation treatments, there was no significant difference between the soybean-tobacco and maize-tobacco treatments. 


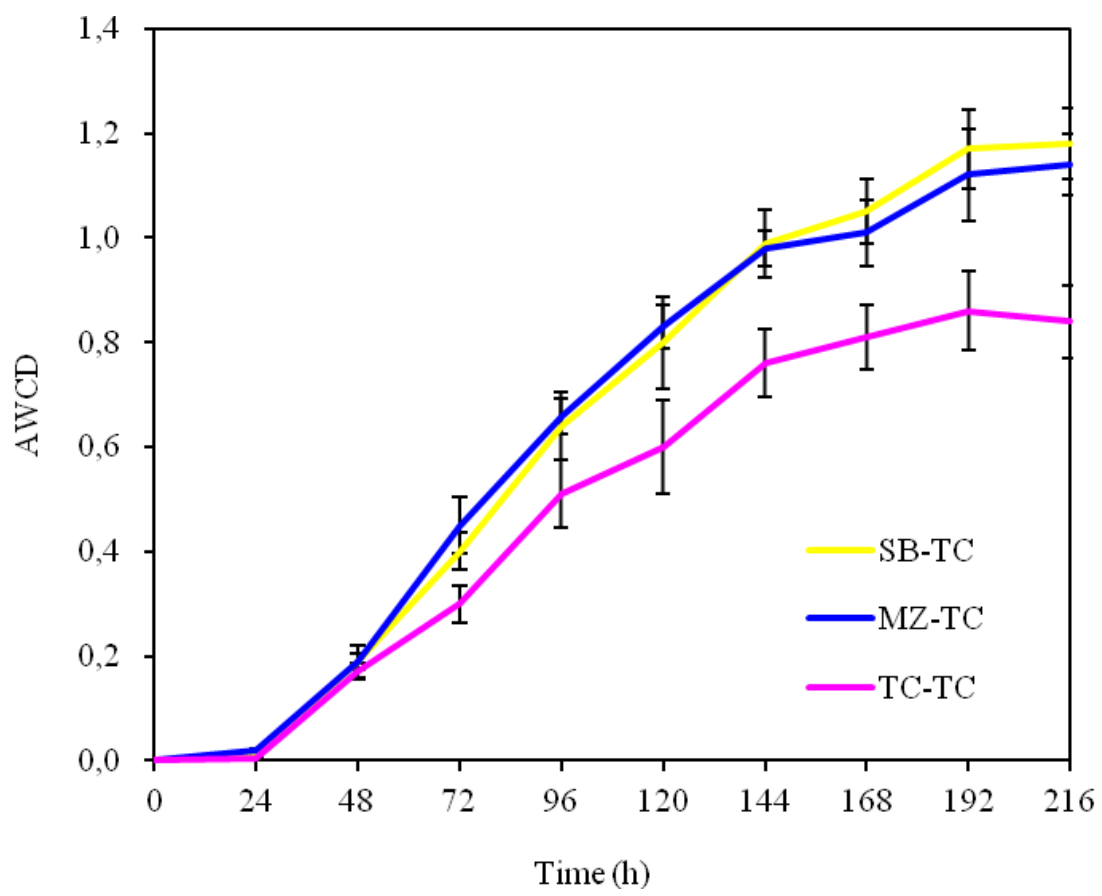

Figure 1. Average well color development $(A W C D)$ obtained by Biolog-Eco Plate ${ }^{T M}$ incubation of all treatments. SB-TC, soybean-tobacco; MZ-TC, maize-tobacco; and TC-TC, tobacco monocropping

Table 1. Bacterial diversity indices of soils based on community level physiological profile. Treatment: SB-TC, soybean-tobacco; MZ-TC, maize-tobacco; and TC-TC, tobacco monocropping. Different letters indicate significant difference at $P<0.05$

\begin{tabular}{c|c|c|c|c}
\hline Treatment & $\begin{array}{c}\text { Shannon-Wiener } \\
\text { index }(\boldsymbol{H})\end{array}$ & $\begin{array}{c}\text { Simpson-diversity } \\
\text { index }(\boldsymbol{D})\end{array}$ & $\begin{array}{c}\text { Substrate } \\
\text { richness }(\boldsymbol{S})\end{array}$ & $\begin{array}{c}\text { McIntosh } \\
\text { index }(\boldsymbol{U})\end{array}$ \\
\hline SB-TC & $1.31 \pm 0.01 \mathrm{a}$ & $0.94 \pm 0.00 \mathrm{a}$ & $23.33 \pm 0.58 \mathrm{a}$ & $4.71 \pm 0.57 \mathrm{a}$ \\
MZ-TC & $1.32 \pm 0.05 \mathrm{a}$ & $0.94 \pm 0.01 \mathrm{a}$ & $24.33 \pm 1.53 \mathrm{a}$ & $4.64 \pm 1.18 \mathrm{a}$ \\
TC-TC & $1.24 \pm 0.03 \mathrm{~b}$ & $0.92 \pm 0.01 \mathrm{~b}$ & $21.33 \pm 0.58 \mathrm{~b}$ & $4.97 \pm 0.62 \mathrm{a}$ \\
\hline
\end{tabular}

\section{Principal component analysis of bacterial community}

Microbial consumption of polymers, amino acids, amines/amides and miscellaneous compounds were significantly higher in soybean-tobacco rotation soils compared to that found in tobacco monocropping soil (Fig. 2). On the contrary, the consumption of carbohydrates was lower in soybean-tobacco soils than in tobacco monocropping soil. However, similarly to soybean-tobacco rotation treatment, soil microbial communities of maize-tobacco rotation treatment showed large polymers utilization.

The principal component analysis (PCA) of bacterial substrate utilization patterns (Fig. 3) explained $79.42 \%$ of the total variance, with the first principle component having the greatest power of separation $(70.21 \%)$. The PCA score plot revealed that the soybean-tobacco and maize-tobacco rotation treatments were clustered together and separated from the tobacco monocropping treatment. 


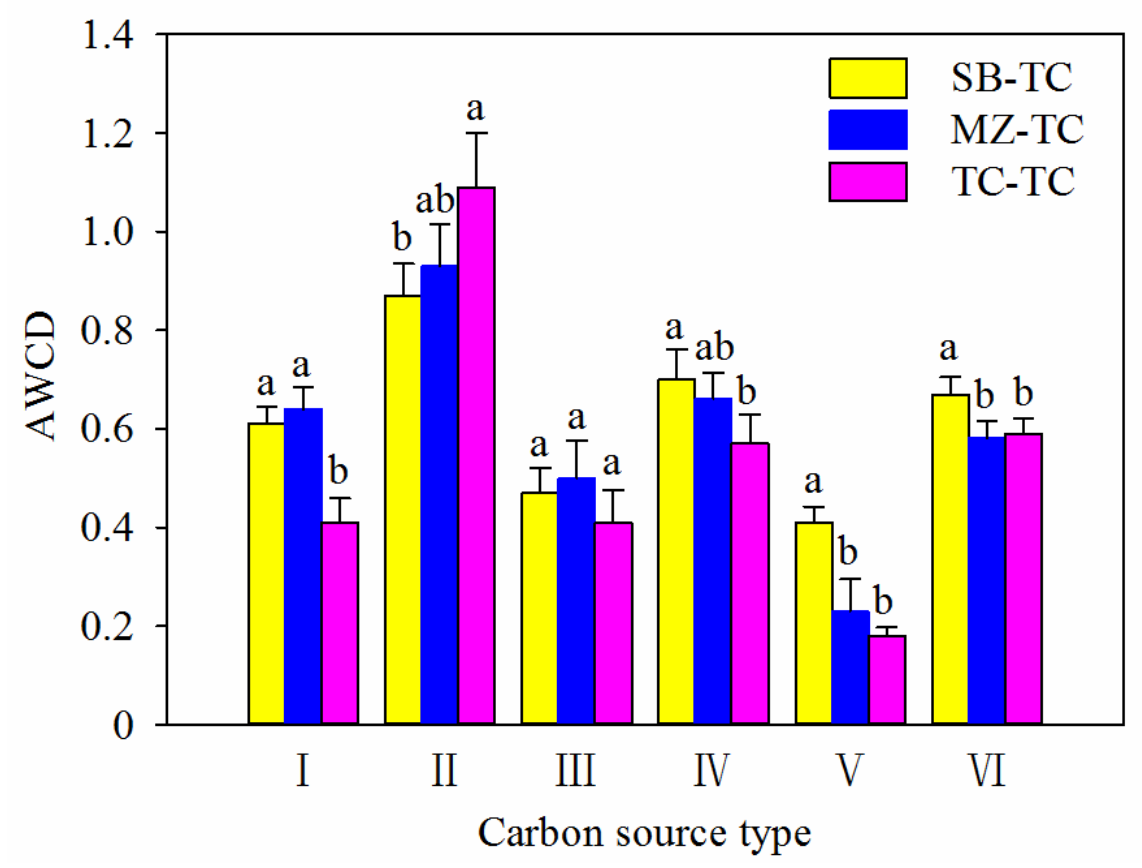

Figure 2. Relative use efficiency of soil microbial community on different carbon sources. I :Polymers; II: Carbohydrate; III Carboxylic acids; IV: Amino acids; V: Amines/amides; VI Miscellaneous. SB-TC, soybean-tobacco; MZ-TC, maize-tobacco; and TC-TC, tobacco monocropping. Different letters indicate significant difference at $P<0.05$

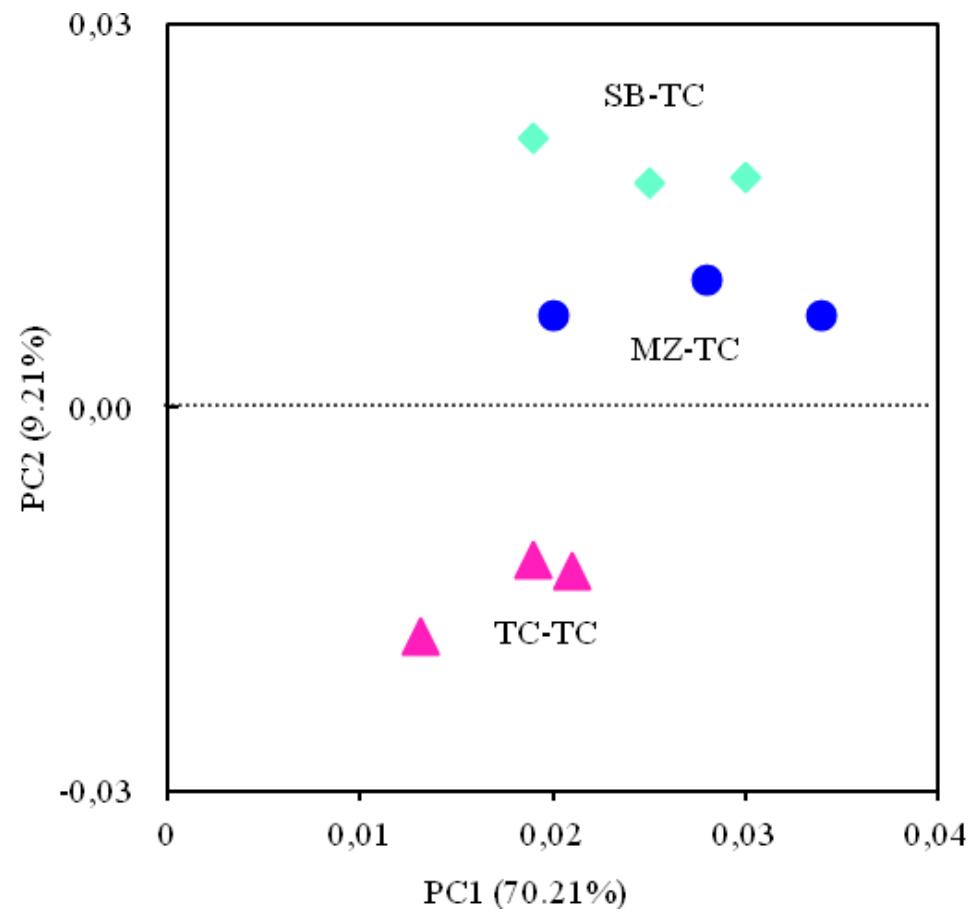

Figure 3. Principal component analysis (PCA) of the use on different carbon sources for soil microbial community. SB-TC, soybean-tobacco; MZ-TC, maize-tobacco; and TC-TC, tobacco monocropping 


\section{Bacterial community analysis using pyrosequencing}

In this study, we assessed and compared the composition of soil bacterial communities derived from the soybean-tobacco, maize-tobacco and tobacco monocropping libraries through the pyrosequencing-based analysis of 16S rRNA gene sequences. Pyrosequencing analysis of the V1-V3 region of the $16 \mathrm{~S}$ rRNA genes resulted in 57,084 high-quality sequences reads with a read length of $\geq 300 \mathrm{bp}$ across all samples. The average read length was $434 \mathrm{bp}$. The read numbers were uneven per sample, ranging from 18,343 to 19,911, with an average of 19,028 (Table 2). All samples were randomly reduced to the same size using MOTHUR based on the sample with the smallest number of reads.

Table 2. Number of $16 S$ rRNA gene sequences derived from three libraries. Treatment: SB-TC, soybean-tobacco; MZ-TC, maize-tobacco; and TC-TC, tobacco monocropping

\begin{tabular}{c|c|c|c}
\hline Treatment & No. obtained sequences $\geq \mathbf{4 0 0}$ bp & Bases (bp) & Average length (bp) \\
\hline SB-TC & 18,343 & $5,934,166$ & 426.2 \\
MZ-TC & 19,911 & $6,243,854$ & 436.3 \\
TC-TC & 18,830 & $6,091,675$ & 439.7 \\
\hline
\end{tabular}

\section{Bacterial alpha-diversity}

To determine rarefaction curves and other measures of diversity, OTUs (operational taxonomic units) were identified at 3\% genetic distance. The rarefaction curves indicated consistent differences among the three libraries (Fig. 4). At 3\% genetic distance, the rarefaction curves suggested that the sequencing effort was not large enough to capture the complete diversity of these communities, as the curves did not reach the asymptote with increasing sample size. The same conclusion was observed in terms of coverage (Table 3). The coverage from the three libraries was below 95\%, indicating that the sequencing reads were not sufficient for this analysis.

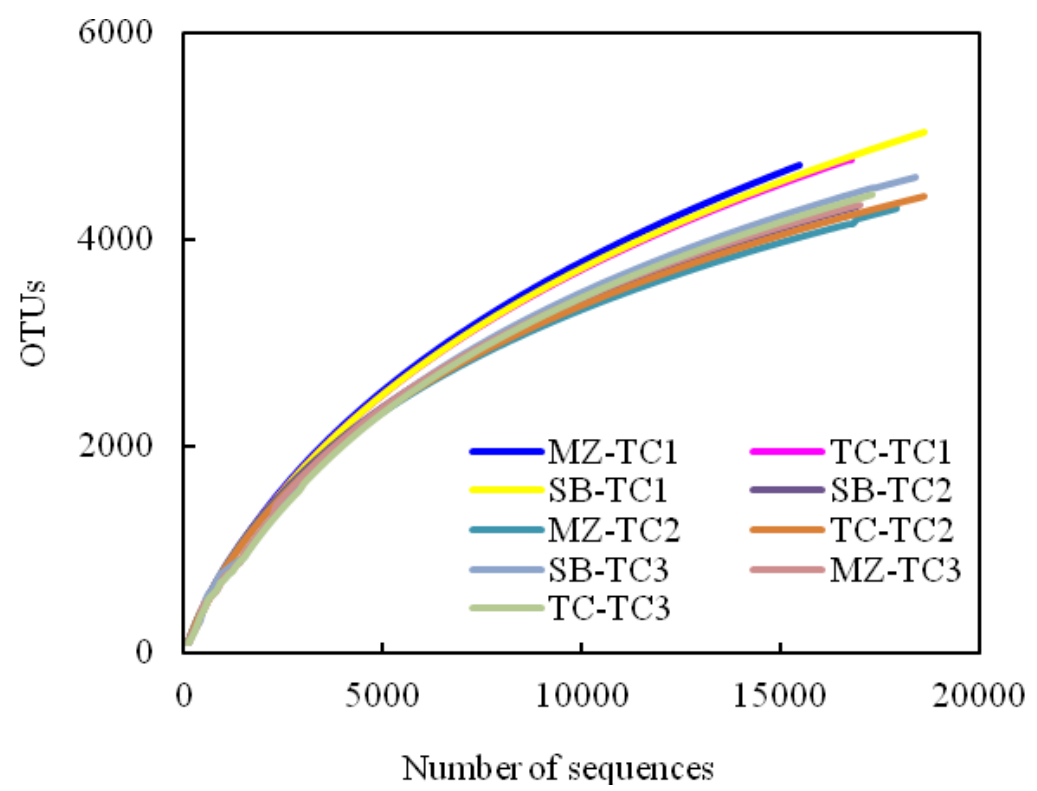

Figure 4. Rarefaction curves indicating the observed number of OTUs at genetic distances of $3 \%$ in all libraries. SB-TC, soybean-tobacco; MZ-TC, maize-tobacco; and TC-TC, tobacco monocropping 
Table 3. Sequencing information and diversity estimated for three samples obtained by 454 pyrosequencing. Treatment: SB-TC, soybean-tobacco; MZ-TC, maize-tobacco; and TC-TC, tobacco monocropping

\begin{tabular}{c|c|c|c|c}
\hline \multirow{2}{*}{ Treatment } & \multicolumn{4}{|c}{ 3\% Genetic distance } \\
\cline { 2 - 5 } & Ace & Chao1 & Shannon & Coverage (\%) \\
\hline SB-TC & 8,806 & 7,221 & 7.78 & 86.6 \\
MZ-TC & 9,812 & 7,892 & 7.91 & 86.3 \\
TC-TC & 7,589 & 7,563 & 7.86 & 86.9 \\
\hline
\end{tabular}

Comparing the mean Shannon diversity index of the three libraries revealed that the highest bacterial diversity at the analyzed genetic distances was found in the maize-tobacco soil, followed by the tobacco monocropping and soybean-tobacco soils. The Chao1 index varied among the three libraries, although not significantly. In addition, the Ace index increased significantly in tobacco rotations soils compared to the tobacco monocropping soil.

\section{Distribution of bacterial composition at the phylum level}

The 57,084 classifiable sequences were affiliated with 15 bacterial phyla (Fig. 5). The dominant phyla across all samples were Proteobacteria, Acidobacteria, Actinobacteria, Chloroflexi, Gemmatimonadetes, and Bacteroidetes, representing 28.10, 18.90, 17.50, $11.00,7.59$, and $5.47 \%$, respectively, of all sequences that were classified below the domain level. These dominant bacterial phyla were found in all samples (Fig. 6). Other sequences belonged to Nitrospirae, Armatimonaddetes, Firmicutes, Fibrobacteres, Chlorobi, Verrucomicrobia and other bacteria, and they were always found in very low proportions $(<5 \%)$.

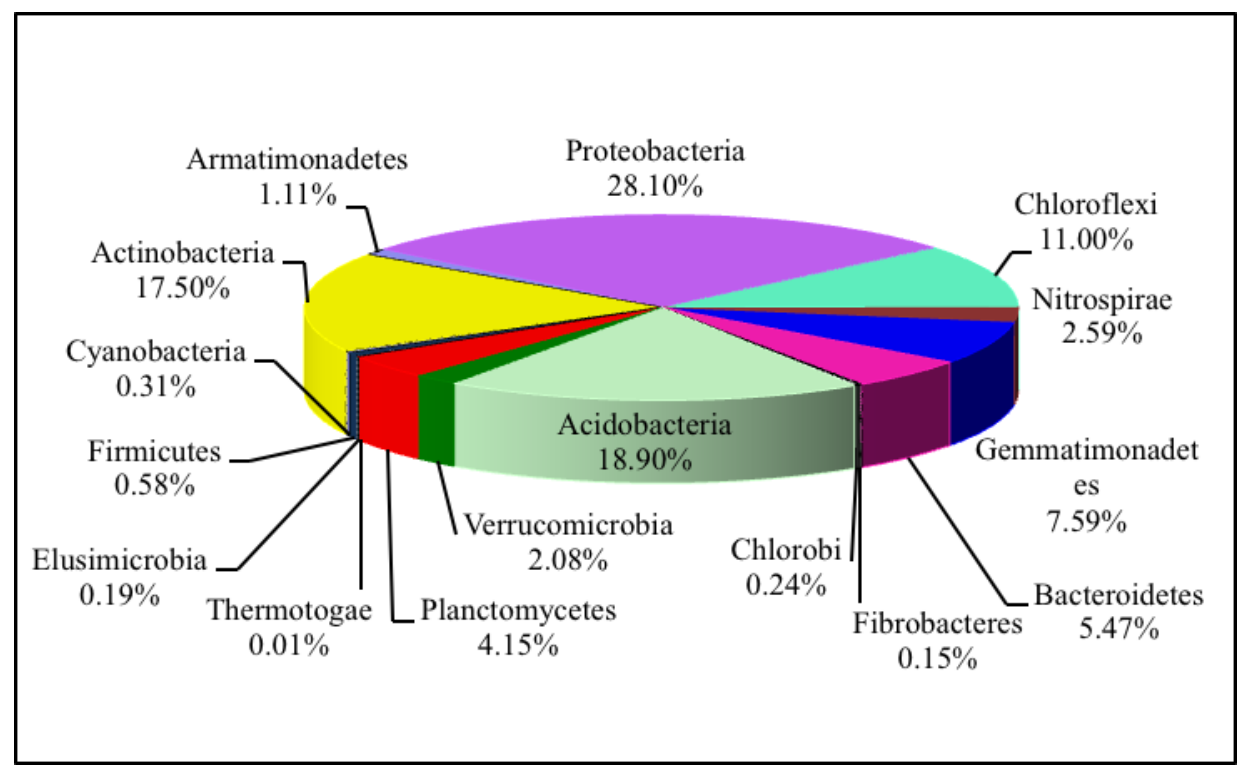

Figure 5. Proportional distribution of different phyla. 


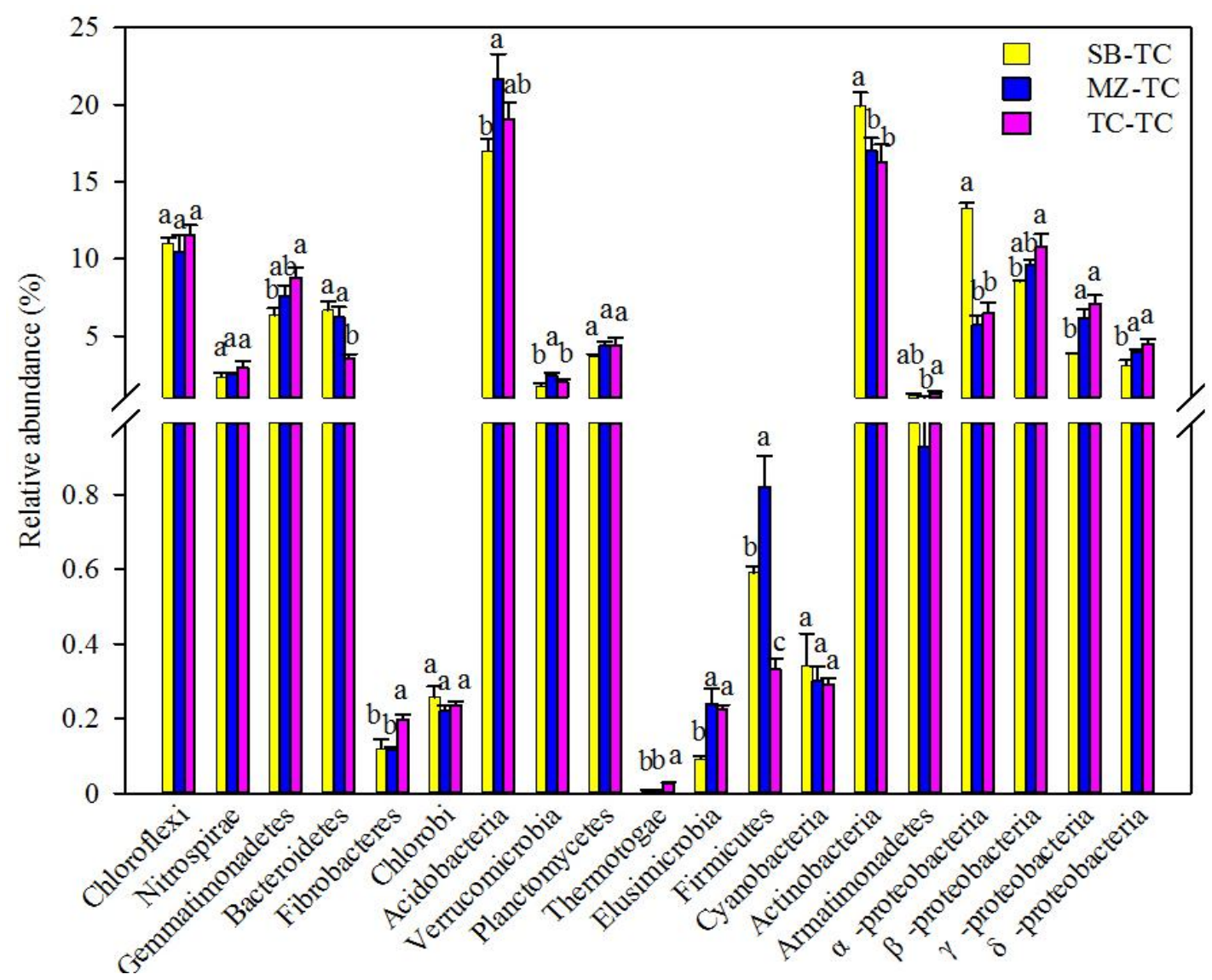

Figure 6. Relative abundance of bacterial phyla and classes of Proteobacteria for each soil library. SB-TC, soybean-tobacco; MZ-TC, maize-tobacco; and TC-TC, tobacco monocropping

The bacterial composition at the phylum level differed between the rotations (soybean-tobacco and maize-tobacco) and the monocropping system. The major phyla were shared by all samples, but in different relative abundances (Fig. 6). In the maize-tobacco and tobacco monocropping soils, Proteobacteria and Acidobacteria were the most abundant phyla, representing $26.58 \%, 28.02 \%$ and $20.63 \%, 19.05 \%$, respectively. However, the soybean-tobacco rotation systems were dominated by the phyla Proteobacteria and Actinobacteria, representing $29.20 \%$ and $19.91 \%$, respectively.

Among the Proteobacteria, $\alpha-, \beta$-, $\gamma$-and $\delta$-proteobacteria were found in all soil libraries (Fig. 6). In the maize-tobacco and tobacco monocropping systems, $\beta$-proteobacteria showed the highest relative abundance $(9.74 \%$ and $10.48 \%)$, followed by $\gamma$-proteobacteria $(6.96 \%$ and $6.89 \%)$, $\alpha$-proteobacteria $(5.82 \%$ and $6.26 \%)$ and $\delta$-proteobacteria $(4.06 \%$ and $4.39 \%)$. The insignificant differences between the maize-tobacco and tobacco monocropping soils indicated that maize as a pre-crop did not significantly impact Proteobacteria in subsequent tobacco soil $(P>0.05)$. Compared with the tobacco monocropping system, Proteobacteria showed significant differences in the soybean-tobacco rotation soil $(P<0.05)$. The relative abundances of Proteobacterial sequences were $13.57 \%$ for $\alpha$-proteobacteria, $8.67 \%$ for $\beta$-proteobacteria, $3.92 \%$ for $\gamma$-proteobacteria and $3.04 \%$ for $\delta$-proteobacteria.

Considering the bacterial distribution in tobacco rotations and monocropping systems, 
variations in relative abundances were also observed for the phyla Acidobacteria, Actinobacteria, Gemmatimonadetes and Bacteroidetes (Fig. 6). The Acidobacteria showed relative abundances of $19.05 \%$ in tobacco monocropping soil, $16.98 \%$ in the soybean-tobacco rotation soil, and $20.63 \%$ in the maize-tobacco rotation soil. A statistical comparison across soil libraries revealed that the relative abundances of Bacteroidetes in soybean-tobacco and maize-tobacco rotation soils, amounting to $6.62 \%$ and $6.23 \%$, respectively, were significantly higher than in tobacco monocropping soil $(3.56 \%)(P<0.05)$. In contrast, the relative abundance of Gemmatimonadetes revealed a significant decrease in the soybean-tobacco $(6.33 \%)$ rotation soils compared to the tobacco monocropping soil $(8.80 \%)(P<0.05)$. Among the Actinobacteria, we observed no significant differences between the soybean-tobacco $(19.91 \%)$ and maize-tobacco $(17.02 \%)$ soils $(P>0.05)$.

\section{Distribution of bacterial composition at the genus level}

Hierarchically clustered heatmap analysis based on 100 predominant bacterial communities at the genus level was used to identify the different compositions of these soil libraries (Fig. 7). Variations at the genus level were observed between soil from tobacco rotations and tobacco monocropping soils. Overall, among the Proteobacteria, the genera Rhodoplanes $(0.41 \%)$, Phenylobacterium $(0.53 \%)$ and Sphingobium $(0.86 \%)$ revealed a significant increase in soybean-tobacco rotation soil compared to the corresponding genera in the tobacco monocropping soil $(0.13 \%, 0.23 \%$ and $0.18 \%$, respectively). In contrast, the relative abundances of Nitrosomonadaceae $(1.90 \%$ and $2.83 \%)$, Sorangium (0.22\% and $0.30 \%)$, Lysobacter $(0.4 \%$ and $0.33 \%)$ and Arenimonas $(0.12 \%$ and $0.25 \%)$ were lower in soybean-tobacco and maize-tobacco soils than in tobacco monocropping soil $(3.29 \%, 0.36 \%, 0.60 \%$ and $0.32 \%$, respectively). The phylum Actinobacteria, which contains the genera Solirubrobacter, Aeromicrobium and Microbacterium, showed significant abundance differences between tobacco rotations and tobacco monocropping soils. Aeromicrobium showed a higher relative abundance in the soybean-tobacco soil, and Solirubrobacter was present at lower relative abundances in the soybean-tobacco and maize-tobacco soils compared with the tobacco monocropping soil. The comparison of the relative abundances of Solirubrobacter revealed no significant difference between the soybean-tobacco and maize-tobacco soils. Within the Bacteroidetes and Acidobacteria, representatives of the genera Adhaeribacter and Candidatus_Chloracidobacterium were variational in the three libraries. Their relative abundances were significantly higher in the soybean-tobacco and maize-tobacco soils than in tobacco monocropping soil. Other genera, Bacillaceae_Bacillus (affiliated with Firmicutes), Zavarzinella (affiliated with Planctomycetes) and Anaerolineaceae (affiliated with Chloroflexi), showed higher relative abundances in the maize-tobacco soil compared to tobacco monocropping soil. 


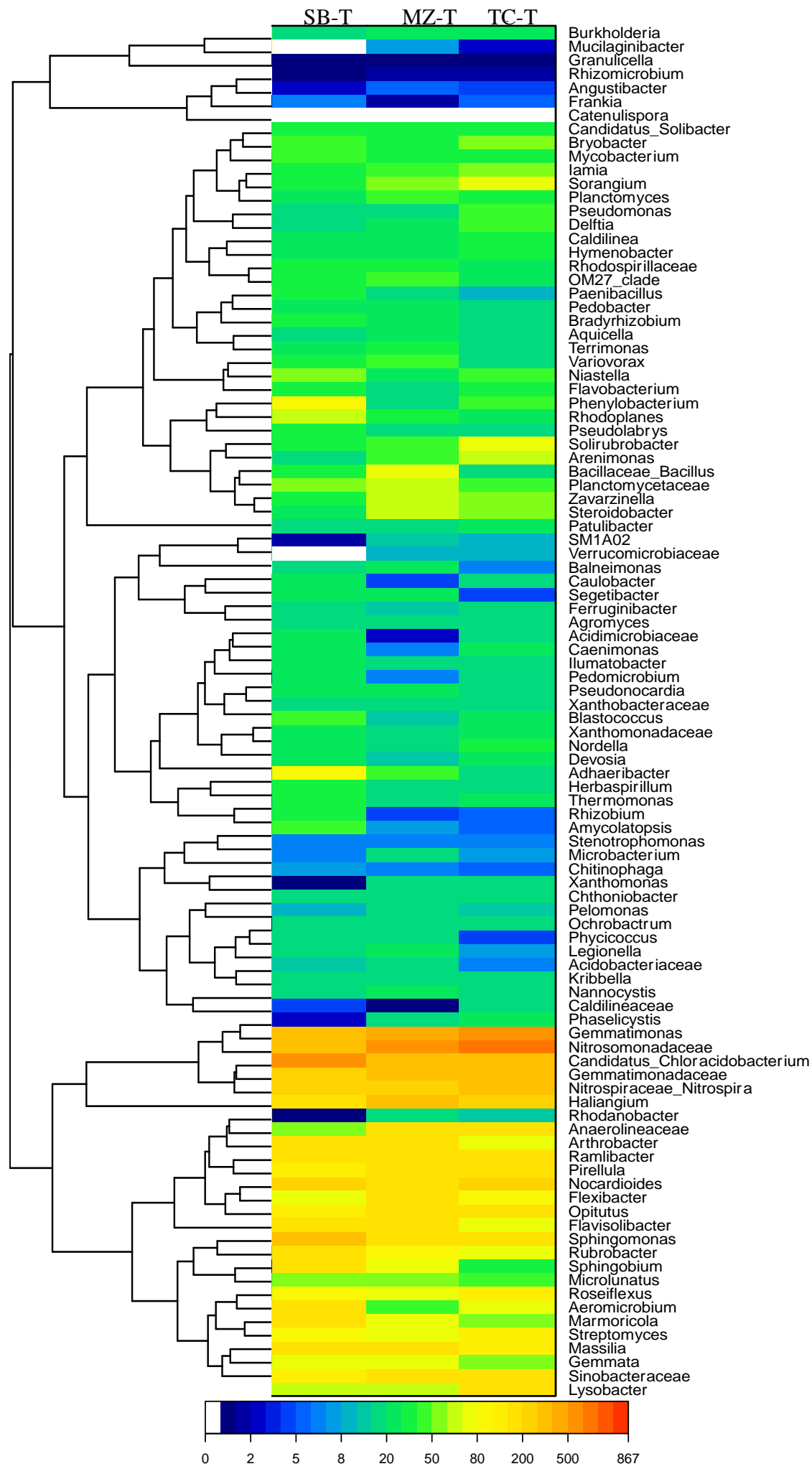

Figure 7. Hierarchical cluster analysis of 100 predominant bacterial communities among the three samples. The Y-axis is the clustering of the most abundant OTUs (5\% distance) in reads. The OTUs were ordered by genus. Sample communities were clustered based on complete linkage method. The color intensity of scale indicates relative abundance of each OTU read. Relative abundance was defined as the number of sequences affiliated with that OTU divided by the total number of sequences per sample 


\section{Discussion}

The residue of the preceding crops has been found to strongly influence soil characteristics, including physic-chemical properties and enzyme activities, which are important for subsequent crops (Urbatzka et al., 2009; Preissel et al., 2015). Numerous bacteria have been known to flourish in the rhizosphere, which is mostly due to the supply of nutrients and the platform supplied by plant residues (Mendes et al., 2013; Bakker et al., 2012; Sugiyama and Yazaki, 2012). In this study, the rhizosphere bacterial communities for the tobacco crop (with pre-crops of soybean, maize and tobacco) were studied using both culture-dependent physiological profiling (Biolog-Eco) and culture-independent 16S rRNA metagenomics (454 pyrosequencing). Using the Biolog-Eco plates, we found that the bacterial activity and diversity in soil from tobacco rotations (soybean and maize) were higher than in the tobacco monocropping soil (Fig. 1 and Table 1). This result was consistent with the findings of Yue (2013) that continuous monocropping in tobacco cultivation led to a decrease in soil bacterial abundance. Meanwhile, some studies have found that rotation systems stimulate soil microbial biomass, enzyme activities and functional diversity and abundance in the soil community structure (Orr et al., 2012; Insam et al., 2015; Plaza et al., 2004). Thus, we speculated that pre-crops of soybean and maize affected the rhizospheric bacterial communities of subsequent tobacco. Moreover, the results of principal component analysis suggested that the soybean-tobacco and maize-tobacco rotation treatments had similar rhizospheric bacterial communities, whereas the continuous cropping of tobacco showed different bacterial communities. The preceding crop effect of grain legumes providing nitrogen has been demonstrated (Hauggaard-Nielsen et al., 2009). As a consequence, this effect varies the bacterial communities for subsequent crops in the soil. In addition to legumes, many broad-leaved crops or summer cereals produce similar rotational benefits (Preissel et al., 2015). The bacterial activity and diversity data showed no significant differences between the soybean-tobacco and maize-tobacco rotation systems, although maize and soybean leave different residues in the field after the crops have been harvested. These results suggested that the plant species planted in the current year was more important in determining the bacterial community in soils than the pre-crop species.

The 454 pyrosequencing analyses revealed 57,084 sequences obtained from the three soil libraries in this study, and Proteobacteria, Acidobacteria and Actinobacteria were the most abundant phyla, which generally agreed with several previous studies reporting that these phyla are capable of having various effects on plant health, including both beneficial and pathogenic interactions (Lee et al., 2008; Berendsen et al., 2012; Li et al., 2016). The rarefaction curves for these samples did not reach the asymptote. Therefore, the deeper sequencing may be required to avoid underestimation of microbial diversity in our samples. But the coverage values for all libraries were not too low (i.e. over $C$. $85 \%$ ), and provide a basis for comparison with other studies. Indeed, our coverage values were largely over reported in oil-contaminated sediment $(\mathrm{C}=61 \%-83 \%)$. Our rarefaction curves based on 454 pyrosequencing did not saturate but we consider, on the basis of the above comparisons, that the majority of the bacterial diversity in our samples was correctly sampled.

Significant differences in bacterial community composition systems were observed between tobacco rotations and tobacco monocropping. The largest shifts in relative abundance were found for Proteobacteria and Acidobacteria. The increase in the relative abundance of $\alpha$-proteobacteria in the soybean-tobacco rotation soil was driven by the increases in the genera Rhodoplanes, Sphingobium and Phenylobacterium (Fig. 7 ), suggesting that these genera are enriched by soybean as a pre-crop. The $\mathrm{pH}$ was the highest in the soybean-tobacco (7.02) soil compared to the tobacco monocropping (6.60) 
and maize-tobacco (6.87) soils. Previous research has demonstrated that $\alpha$-proteobacteria and $\gamma$-proteobacteria showed positive relationships to soil $\mathrm{pH}$ (Rousk et al., 2010). The phylum Chloroflexi is ubiquitous in various soil samples, and its relative abundance was frequently higher than the abundance of Bacteroidetes (Roesch et al., 2007). This finding was further supported by the changes in our study. The relative abundance of Acidobacteria increased significantly in the maize-tobacco rotation soil compared to the soybean-tobacco soil. The abundance of the phylum Acidobacteria correlates with the soil pH (Hartman et al., 2008; Jones et al., 2009), and acidobacterial subgroups 1 and 2 decreased with increasing $\mathrm{pH}$ (Lauber et al., 2009), which is in consistent to our study. The biological functions of Acidobacteria are not well known because most microorganisms of this phylum have not been cultured (Yamada and Sekiguchi, 2009). However, as recent study found that Acidobacteria are capable of degrading plant litter in soils (Eichorst et al., 2011), the presence of maize residues in the maize-tobacco rotation soil may have contributed to the observed differences. Symbiotically fixed N may contribute to the nutrient availability, soil structure, and microbial activities of subsequent crops in rotation via the $\mathrm{N}$ mineralization of plant residues (Urbatzka et al., 2009). The relative abundance of Actinobacteria was strongly shaped by the pre-crop, and a significant increase was observed in the soybean-tobacco rotation soil compared to the tobacco monocropping soil. Actinobacteria members play an important role in the degradation of recalcitrant compounds and prevent infection with pathogenic microorganisms by secreting various antibiotics (Nour et al., 2003). In addition, previous studies have demonstrated that the Bacillaceae_Bacillus genus (affiliated with Firmicutes) was primarily involved in biological control against different soil-borne pathogens (Pal et al., 2004). Since the Bacillaceae_Bacillus genus showed higher relative abundances in soil from tobacco rotations with (soybean and maize) than in tobacco monocropping soil. The higher abundance of Bacillaceae_Bacillus in the tobacco rotation systems may contribute to disease suppression, and soybean as a pre-crop resulted in the highest bacterial community diversity and activity compared with maize and tobacco. Such results suggest that the soybean-tobacco rotation system, from a biological perspective, may represent a good strategy for enhancing microbial diversity and maintaining soil biological fertility.

\section{Conclusion}

Our research shows that the bacterial communities in the soybean-tobacco and maize-tobacco rotation soils are diverse, and various members can apparently be attributed to the pre-crops (compared to the corresponding bacterial communities in the tobacco monocropping system soil). The results obtained from the present field investigations, by using Biolog-Eco platess and next-generation sequencing from the three treatments, showed that (i) the bacterial activity and diversity in soil from tobacco rotations (soybean and maize) were higher than in tobacco monocropping soil; (ii) there were increased relative abundances of Actinobacteria and Bacteroidetes, particularly of the genera of Rhodoplanes, Adhaeribacter, and Bacillaceae_Bacillus in the soybean-tobacco and maize-tobacco rotation soils compared with the tobacco monocropping soil. 
Acknowledgements. This research was supported by the "Twelfth Five-Year" National Science and Technology Support Program of China (2011BAD08B02-3), the Major Project for Heilongjiang Province Science and Technology Program (GZ13B004), and the Fundamental Research Funds for the Central Universities (2572014AA16).

Conflict of interest. The author confirms that this article content has no conflict of interest.

\section{REFERENCES}

[1] Bakker, M. G., Manter, D. K., Sheflin, A. M., Weir, T. L., Vivanco, J. M. (2012): Harnessing the rhizosphere microbiome through plant breeding and agricultural management. - Plant \& Soil 360:1-13.

[2] Berendsen, R. L., Pieterse, C. M. J., Bakker, P. A. H. M. (2012): The rhizosphere microbiome and plant health. - Trends in Plant Science 17:478-486.

[3] Dick, R. P. (1992): A review: long-term effects of agricultural systems on soil biochemical and microbial parameters. - Agriculture Ecosystems \& Environment 40:25-36.

[4] Eichorst, S. A., Kuske, C. R., Schmidt, T. M. (2011): Influence of plant polymers on the distribution and cultivation of bacteria in the phylum Acidobacteria. - Applied and Environment Microbiology 77:586-596.

[5] Garland, J. L. (1996): Analytical approaches to the characterization of samples of microbial communities using patterns of potential C source utilization. - Soil Biology \& Biochemistry 28:213-221.

[6] Hartman, W. H., Richardson, C. J., Vilgalys, R., Bruland, G. L. (2008): Environmental and anthropogenic control of bacterial communities in wetland soils. - Proceedings of the National Academy of Sciences of the United States of America 105:17842-17847.

[7] Hauggaard-Nielsen, H., Mundus, S., Jensen, E. S. (2009): Nitrogen dynamics following grain legumes and subsequent catch crops and the effects on succeeding cereal crops. Nutrient Cycling Agroecosystems 84:281-291.

[8] Hecht, S. S., Castonguay, A., Chung, F. L., Hoffmann, D. (1984): Carcinogenicity and metabolic activation of tobacco-specific nitrosamines: current status and future prospects. Iarc Scientific Publications 17:763-778.

[9] Insam, H., Gómez-Brandón, M., Ascher, J. (2015): Manure-based biogas fermentation residues-Friend or foe of soil fertility? - Soil Biology \& Biochemistry 84:1-14.

[10] Jones, R. T., Robeson, M. S., Lauber, C. L., Hamady, M., Knight, R., Fierer, N. (2009): A comprehensive survey of soil acidobacterial diversity using pyrosequencing and clone library analyses. ISME Jouranl 3:442-453.

[11] Kirkegaard, J., Christen, O., Krupinsky, J., Layzell, D. (2008): Break crop benefits in temperate wheat production. - Field Crops Research 107:185-195.

[12] Lauber, C. L., Hamady, M., Knight, R., Fierer, N. (2009): Pyrosequencing-based assessment of soil $\mathrm{pH}$ as a predictor of soil bacterial community structure at the continental scale. - Applied and Environment Microbiology 75:5111-5120.

[13] Lee, S., Ka, J., Cho, J. (2008): Members of the phylum Acidobacteria are dominant and metabolically active in rhizosphere soil. - FEMS Microbiology Letters 285:263-269.

[14] Li, C., Li, X., Kong, W., Wu, Y., Wang, J. (2010): Effect of monoculture soybean on soil microbial community in the Northeast China. - Plant \& Soil 330:423-433.

[15] Li, X., Sun, M., Zhang, H., Xu, N., Sun, G. (2016): Use of mulberry-soybean intercropping in salt-alkali soil impacts the diversity of the soil bacterial community. -Microbial Biotechnology 9:293-304.

[16] Lienhard, P., Tivet, F., Chabanne, A., Dequiedt, S., Lelievre, M., Sayphoummie, S., Leudphanane, B., Chemidlin, N., Boure, N. P., Seguy, L., Maron, P., Ranjard, L. (2013): No-till and cover crops shift soil microbial abundance and diversity in Laos tropical 
grasslands. - Agronomy for Sustainable Development 33:375-384.

[17] Mendes, R., Garbeva, P., Raaijmakers, J. M. (2013): The rhizosphere microbiome: significance of plant beneficial, plant pathogenic, and human pathogenic microorganisms. FEMS Microbiology Reviews 37:634-663.

[18] Morari, F., Lugato, E., Berti, A., Giardini, F. (2006): Long-term effects of recommended management practices on soil carbon changes and sequestration in north-eastern Italy. Soil Use \& Management 22:71-81.

[19] Nannipieri, P., Ascher, J., Ceccherini, M. T., Landi, L., Pietramellara, G., Renella, G. (2003): Microbial diversity and soil functions. - European Journal of Soil Science 54:655-670.

[20] Nazih, N., Finlay-Moore, O., Hartel, P. G., Fuhrmann, J. J. (2001): Whole soil fatty acid methyl ester (FAME) profiles of early soybean rhizosphere as affected by temperature and matric water potential. - Soil Biology and Biochemistry 33:693-696.

[21] Nour, S. M., Lawrence, J. R., Zhu, H., Swerhone, G. D. W., Welsh, M., Welacky, T. W., Topp, E. (2003): Bacteria associated with cysts of the soybean cyst nematode (Heterodera glycines). - Applied and Environment Microbiology 69:607-615.

[22] Orr, C. H., Leifert, C., Cummings, S. P., Cooper, J. M. (2012): Impacts of organic and conventional crop management on diversity and activity of free-living nitrogen fixing bacteria and total bacteria are subsidiary to temporal effects. - Plos One 7:483-496.

[23] Pal, H., Ray, B. F., Vivanco, J. M. (2004): Biocontrol of Bacillus subtilis against infection of Arabidopsis roots by Pseudomonas syringae is facilitated by biofilm formation and surfactin production. - Plant Physiology 134:307-319.

[24] Plaza, C., Hernández, D., García-Gil, J. C., Polo, A. (2004): Microbial activity in pig slurry-amended soils under semiarid conditions. - Soil Biology \& Biochemistry 36:1577-1585.

[25] Ponge, J. F., Pérèset, G., Guernion, M., Ruiz-Camacho, N., Cortet, J., Pernin, C., Villenave, C., Chaussod, R., Laurent, F. M., Bispo, A., Cluzeau, D. (2013): The impact of agricultural practices on soil biota: a regional study. - Soil Biology \& Biochemistry 67:271-284.

[26] Preissel, S., Reckling, M., Schläfke, N., Zander, P. (2015): Magnitude and farm-economic value of grain legume pre-crop benefits in Europe: A review. - Field Crops Research 175:64-79.

[27] Qiu, M. H., Zhang, R. F., Xue, C., Zhang, S. S., Li, S. Q., Zhang, N., Shen, Q. R. (2012): Application of bio-organic fertilizer can control Fusarium wilt of cucumber plants by regulating microbial community of rhizosphere soil. - Biology \& Fertility of Soils 48:807-816.

[28] Quadros, P. D. D., Kateryna, Z., Austin, D. R. (2012): The effect of tillage system and crop rotation on soil microbial diversity and composition in a subtropical acrisol. - Diversity 4:375-395.

[29] Roesch, L. F., Fulthorpe, R. R., Riva, A., Casella, G., Hadwin, A. K., Kent, A. D., Daroub, S. H., Camargo, F. A., Farmerie, W. G., Triplett, E. W. (2007): Pyrosequencing enumerates and contrasts soil microbial diversity. - Isme Journal 1:283-290.

[30] Rousk, J., Bååth, E., Brookes, P. C., Lauber, C. L., Lozupone, C., Caporaso, J. G., Knight, R., Fierer, N. (2010): Soil bacterial and fungal communities across a $\mathrm{pH}$ gradient in an arable soil. - Isme Journal 4:1340-1351.

[31] Rutgers, M., Breure, A. M., Insam, H., Bloem, J., Benedetti, A., Hopkins, D. W. (2006): Substrate utilization in Biolog ${ }^{\mathrm{TM}}$ plates for analysis of CLPP. - Wallington, CABI Publishing 212-227.

[32] Su, C., Lei, L. P., Duan, Y. Q., Zhang, K. Q., Yang, J. K. (2012): Culture-independent methods for studying environmental microorganisms: methods, application, and perspective. - Applied Microbiology Biotechnology 93:993-1003.

[33] Sugiyama, A., Yazaki, K. (2012): Root exudates of legume plants and their involvement in interactions with soil microbes. - Springer Berlin Heidelberg 12:27-48. 
[34] Trasar-Cepeda, C., Leirós, M. C., Seoane, S., Gil-Sotres, F. (2008): Biochemical properties of soils under crop rotation. - Applied Soil Ecology 39:133-143.

[35] Urbatzka, P., Graß, R., Haase, T. (2009): Fate of legume-derived nitrogen in monocultures and mixtures with cereals. - Agriculture Ecosystems \& Environment 132:116-125.

[36] Varvel, G. E. (2008): Soil organic carbon changes in diversified rotations of the Western Corn Belt. - Soilence Society of America Journal 70:426-433.

[37] Wright, A. L., Hons, F. M. (2005): Soil carbon and nitrogen storage in aggregates from different tillage and crop regimes. - Soil Science Society of America Journal 69:141-147.

[38] Yamada, T., Sekiguchi, Y. (2009): Cultivation of uncultured chloroflexi subphyla: significance and ecophysiology of formerly uncultured chloroflexi 'subphylum i' with natural and biotechnological relevance. - Microbes and Environments 2:205-216.

[39] Yue, B. B., Li, X., Zhang, H. H., Jin, W. W., Xu, N., Zhu, W. X., Sun, G. Y. (2013): Soil microbial diversity and community structure under continuous tobacco cropping soils. Soils (in Chinese) 45:116-119.

[40] Zarraonaindia, I., Smith, D. P., Gilbert, J. A. (2013): Beyond the genome: community-level analysis of the microbial world. - Biology \& Philosophy 28:261-282. 